\section{Does the prevalence of radiographic hand osteoarthritis in patients with HIV-1 infection increase or not?}

Hand osteoarthritis (HOA) is a heterogeneous disease with the involvement of different joints and varying levels of symptoms. Recent studies showed that the prevalence of HOA varied with gender, age and different hand joints. ${ }^{1-3}$ Different prevalence and longitudinal course of radiographic, erosive and symptomatic HOA were also described in the general population. ${ }^{2}$ Nevertheless, the accurate influence factors of HOA prevalence are still far from well understood. We read with deep interest the article by Anne-Laurence Tomi et $a l^{4}$ related to the prevalence and severity of radiographic HOA in patients with HIV-1 infection associated with metabolic syndrome. This crosssectional Metabolic Syndrome and Fibrosis-Osteoarthritis (METAFIB-OA) study suggested that HOA frequency was greater in patients with HIV-1 infection than the general population, and it was more significant in those with metabolic syndrome (MetS). Thus, the authors thought that HIV-1 infection represents a special promotion for radiographic HOA and that there should be a 'HIV-related OA' subtype beyond classical OA phenotypes. We really appreciate the work that has been done by the authors. However, some worthwhile issues need further exploration.

First of all, this study did not analyse the general risk factors of HOA. As a prevalent disorder, HOA is not one single disease, but a heterogeneous group of disorders. ${ }^{1-3} 5$ The prevalence of HOA showed great variation, which may be due to differences in the types of populations and/or risk factors such as genetic background or environmental exposures across cohorts. ${ }^{2} 56$ To date, studies showed many factors related to HOA, including age, sex, populations, lifestyle, inflammation, bone mineral density, atherosclerosis, cardiovascular disease and metabolic health. ${ }^{1-3}$ 5-10 This study was a cross-sectional single-centre study with only 301 effective patients included in France. On one hand, the sample was too small to exclude any other factors promoting HOA apart from MetS. On the other hand, there might be a huge difference about populations and lifestyle between patients in METAFIB-OA study and the Framingham study of the USA, which was used as general population control group. The authors declared that there was not any available HOA assessment in a population-based cohort in France; so, they used the Framingham study. We agree with the authors' point of view. But these factors might affect the accuracy of their results to a large extent.

Second, one objective of the study was to compare the prevalence in patients with HIV-1 infection with the general population. The authors measured many HIV-1 infection features such as duration of HIV infection, ultrasensitive HIV viral load, duration of exposure to protease inhibitors, CD4 level, CD4/CD8 ratio, plasma high-sensitivity $\mathrm{C}$ reactive protein (hsCRP) level, plasma interleukin 6 level, plasma-soluble CD14 (sCD14) level and plasma-soluble CD163 (sCD163) level, and tried to find their association with HOA. However, no factor related to HOA was found except plasma sCD14 level. What was more, this association became non-significant $(\mathrm{OR}=3.9,95 \%$ CI 0.9 to 17.2; $\mathrm{p}=0.07)$ as well after adjustment for MetS. So it is hard to draw a conclusion that HIV-1 infection was a determinant of HOA. The authors also found that radiographic HOA was significantly more frequent in patients with MetS than those without it $(64.5 \%$ vs $46.3 \%$; $=0.002)$, but similar results were reported recently in general population studies too. ${ }^{9} 10$ The role of HIV-1 infection in this pathological process is still a controversial issue, which needs further research.

Finally, only 36 female patients were included in this study, and the prevalence of HOA had no statistic difference between the METAFIB women and Framingham women. While the study showed there were more than 3.5-fold higher rates on risk of HOA in women aged 50-60 years when compared with men of similar age. ${ }^{1}$ This difference might change as more female patients are being brought into study. But deficiency of female patients might have led to the bias of the results. Besides, two different readers scored the radiographs in the study. However, the interreader concordance for the total Kellgren-Lawrence scale was only 0.64 (95\% CI 0.61 to 0.67 ) as described in the article. This low inter-reader reliability might affect the results. Moreover, according to the materials, HOA prevalence should be $55.5 \%(167 / 301)$ in all patients with HIV-1 infection, while it was $55.6 \%$ in the Abstract; HOA prevalence in HIV-1 positive patients with MetS (HIV-1+MetS+) should be $64.5 \%$ (98/152), while it was $64.7 \%$ and $64.4 \%$ in the Abstract and Table 2, respectively. When considering men within each age group, HOA frequency was $57.8 \%$ $(148 / 256)$ in patients with HIV-1 infection, while it was $55.8 \%$ in the Abstract and Table 3. Diabetes was defined as glycaemia $>6 \mathrm{mmol} / \mathrm{L}$ in Table 1 , but it was defined as glycaemia $>7 \mathrm{mmol} / \mathrm{L}$ in Table 4. These need to be corrected.

We respect the great contributions of the authors, and we would also be very interested in the authors' response regarding the above issues.

\section{Wei Luo, Guang-hua Lei, Hong-bo He, Yu-sheng Li}

Department of Orthopaedics, Xiangya Hospital, Central South University, Changsha, Hunan, China

Correspondence to Dr Yu-sheng Li, Department of Orthopaedics, Xiangya Hospital, Central South University, Xiangya Road, Changsha, Hunan 410008, China; lys0209@163.com

Twitter Follow Hong-bo He at @88569810@qq.com

Contributors WL, Y-sL: concept, writing; G-hL, H-bH: concept, revising.

Funding This work was supported by the Natural Science Foundation of China (grant number 81401838, 81402224).

Competing interests None declared.

Provenance and peer review Not commissioned; internally peer reviewed.

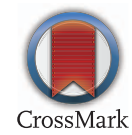

To cite Luo W, Lei G-hua, He H-bo, et al. Ann Rheum Dis 2016;75:e51.

Accepted 29 April 2016

Published Online First 24 May 2016

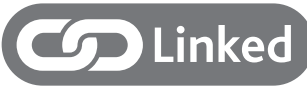

- http://dx.doi.org/10.1136/annrheumdis-2016-209833

Ann Rheum Dis 2016;75:e51. doi:10.1136/annrheumdis-2016-209808

\section{REFERENCES}

1 Prieto-Alhambra D, Judge A, Javaid MK, et al. Incidence and risk factors for clinically diagnosed knee, hip and hand osteoarthritis: influences of age, gender and osteoarthritis affecting other joints. Ann Rheum Dis. 2014;73:1659-64.

2 Haugen IK, Englund M, Aliabadi P, et al. Prevalence, incidence and progression of hand osteoarthritis in the general population: the Framingham Osteoarthritis Study. Ann Rheum Dis. 2011;70:1581-6. 
3 Marshall M, Peat G, Nicholls E, et al. Subsets of symptomatic hand osteoarthritis in community-dwelling older adults in the United Kingdom: prevalence, inter-relationships, risk factor profiles and clinical characteristics at baseline and 3-years. Osteoarthr Cartil. 2013;21:1674-84.

4 Tomi AL, Sellam J, Lacombe K, et al. Increased prevalence and severity of radiographic hand osteoarthritis in patients with HIV-1 infection associated with metabolic syndrome: data from the cross-sectional METAFIB-OA study. Ann Rheum Dis 2016. Published Online First 31 Mar 2016. doi:10.1136/annrheumdis-2016-209262

5 Kloppenburg M, Kwok WY. Hand osteoarthritis-a heterogeneous disorder. Nat Rev Rheumatol 2011;8:22-31.

6 Kalichman L, Li L, Batsevich V, et al. Prevalence, pattern and determinants of radiographic hand osteoarthritis in five Russian community-based samples. Osteoarthr Cartil 2010;18:803-9.
7 Güler-Yüksel M, Bijsterbosch J, Allaart CF, et al. Accelerated metacarpal bone mineral density loss is associated with radiographic progressive hand osteoarthritis. Ann Rheum Dis 2011;70:1625-30.

8 Hoeven TA, Kavousi M, Clockaerts S, et al. Association of atherosclerosis with presence and progression of osteoarthritis: the Rotterdam Study. Ann Rheum Dis 2013;72:646-51.

9 Haugen IK, Ramachandran VS, Misra D, et al. Hand osteoarthritis in relation to mortality and incidence of cardiovascular disease: data from the Framingham heart study. Ann Rheum Dis 2015;74:74-81.

10 Bos SD, Beekman M, Maier AB, et al. Metabolic health in families enriched for longevity is associated with low prevalence of hand osteoarthritis and influences OA biomarker profiles. Ann Rheum Dis 2013;72:1669-74. 